\title{
Comparative Study of CEC'2013 Problem Using Dual Population Genetic Algorithm
}

\author{
A. J. Umbarkar \\ Department of Information Technology, Walchand College of Engineering Sangli, MS, India \\ Email: anantumbarkar@rediffmail.com \\ L. R. Moon \\ Department of Information Technology, Walchand College of Engineering Sangli, MS, India \\ Email: latikmoon@gmail.com \\ P. D. Sheth \\ Department of MCA \\ Government College of Engineering, Karad, 415-124, India \\ Email: pranalisheth@gmail.com
}

Received: 08 December 2017; Accepted: 19 January 2018; Published: 08 September 2018

\begin{abstract}
Evolutionary Algorithms (EAs) are found to be effective for solving a large variety of optimization problems. In this Paper Dual Population Genetic Algorithm (DPGA) is used for solving the test functions of Congress on Evolutionary Computation 2013 (CEC'2013), by using two different crossovers. Dual Population Genetic Algorithm is found to be better in performance than traditional Genetic Algorithm. It is also able to solve the problem of premature convergence and diversity of the population in genetic algorithm. This paper proposes Dual Population Genetic Algorithm for solving the problem regarding unconstrained optimization. Dual Population Genetic Algorithm is used as metaheuristic which is verified against 28 functions from Problem Definitions and Evaluation Criteria for the Congress on Evolutionary Computation 2013 on unconstrained set of benchmark functions using two different crossovers. The results of both the crossovers are compared with each other. The results of both the crossovers are also compared with the existing results of Standard Particle Swarm Optimization algorithm. The Experimental results showed that the algorithm found to be better for finding the solution of multimodal functions of the problem set.
\end{abstract}

Index Terms-Dual Population Genetic Algorithm, DPGA, Genetic Algorithm, GA, Evolutionary Algorithm, EA, Function Optimization, CEC'2013, k-Point Crossover.

\section{INTRODUCTION}

Evolutionary Algorithms (EAs) is widely used for solving optimization problems. EAs are found to be useful from the last few decades to successfully solve the complex problems. Genetic algorithms (GAs) are population based stochastic evolutionary algorithms. It is based on the principal "survival of fittest". In the evolution of populations, GA loses the population diversity and gets trapped in local optima. This problem in EA is called as "premature convergence problem". It especially occurs in solving complex optimization problems, where search space has a lot of peaks and valleys in the fitness map [1].

The proposed solution to this problem in GA is using two populations instead of using only one. DPGA has two populations- main population and the reserve population. The job of the reserve population is to provide additional population diversity to the main population. The information between the main population and the reserve population is exchanged by means of inter-population crossbreeding. The crossbreeding technique helps to solve the problem of premature convergence.

In this paper, the unconstrained optimization problems defined in CEC'2013 are solved. Section II gives a brief literature review of DPGA. Section III describes DPGA with implementation details such as the crossover operators used in the experimentation. Section IV shows the experimental results, comparison of results and discussion. Section V gives conclusions and future scope.

\section{LITERATURE REVIEW}

This section firstly gives the literature of evolution of DPGA and then gives review of EAs, which are experimented on benchmark functions CEC'2013. The work provides a brief literature review on DPGA and the crossover operators used.

DPGA introduced by Park and Ruy in 2006 [1]. [2] gives the details review of DPGA. DPGA consists of two different populations with different evolutionary 
objectives. The objective of the main population is same as that of regular genetic algorithm which targets to optimize the objective function to its minimum or maximum as per required. The purpose of the reserved population is to maintain diversity. In 2007, Park and Ruy propose DPGA-ED [3]. Difference between a simple DPGA and DPGA-ED is that DPGA-ED evolves by itself. Park and Ruy unveiled DPGA2 that uses two reserve populations instead using only one population for providing diversity to the main population.

[4] proposes the approach of adjusting the distance between the main population and reserve population of DPGA. [5] applied DPGA to non-stationary Optimization.

Umbarkar, Joshi and Hong (2014) [6] improves the performance of DPGA by parallelizing it using multithreads. By using this technique they also solve the problem of population diversity and premature convergence.

Zambrano-Bigiarini, Clerc and Rojas (2013) [7] uses Standard Particle Swarm Optimization algorithm to solve the problem set of CEC'2013.

Elsayed, Sarker and Essam [10] applied GA on CEC 2013. [11] accelerate the Particle Swarm Optimization with Diversity-Guided Convergence and Stagnation Avoidance. [12] proposed the Diversity Enhanced Differential Evolution

\section{Dual Population Genetic Algorithm}

DPGA is a variant of GA which consists of two populations, the main population and the reserve population. Both the populations were initialized with random numbers. Individuals of both the populations were evaluated using their own fitness functions. The new generation of each population is obtained by inbreeding between the parents of the same population (Crossover operator), Crossbreeding between the parents of different populations, and the survival selection among the obtained offspring. The fitness function used for the reserve population, evolutionary process of simple DPGA and crossover operator used in it, are described as follows:

\section{A. Crossover Operators}

The main searching operator in this algorithm is the crossover operator while mutation and crossbreed are considered as a variation or diversity operator. In this paper, we have used two crossovers i.e. $k$-point crossover and Discrete TPX [8]. To provide multiple combinations of selected parents it selects more than one crossover points.

\section{A.1 k-point Crossover Operators}

The $k$-point crossover randomly selects $k$ crossover points cp1 to cpk-1 in the selected parents. Two offspring are created by combining the parents at crossover points. The algorithm for $k$-point crossover is given below:

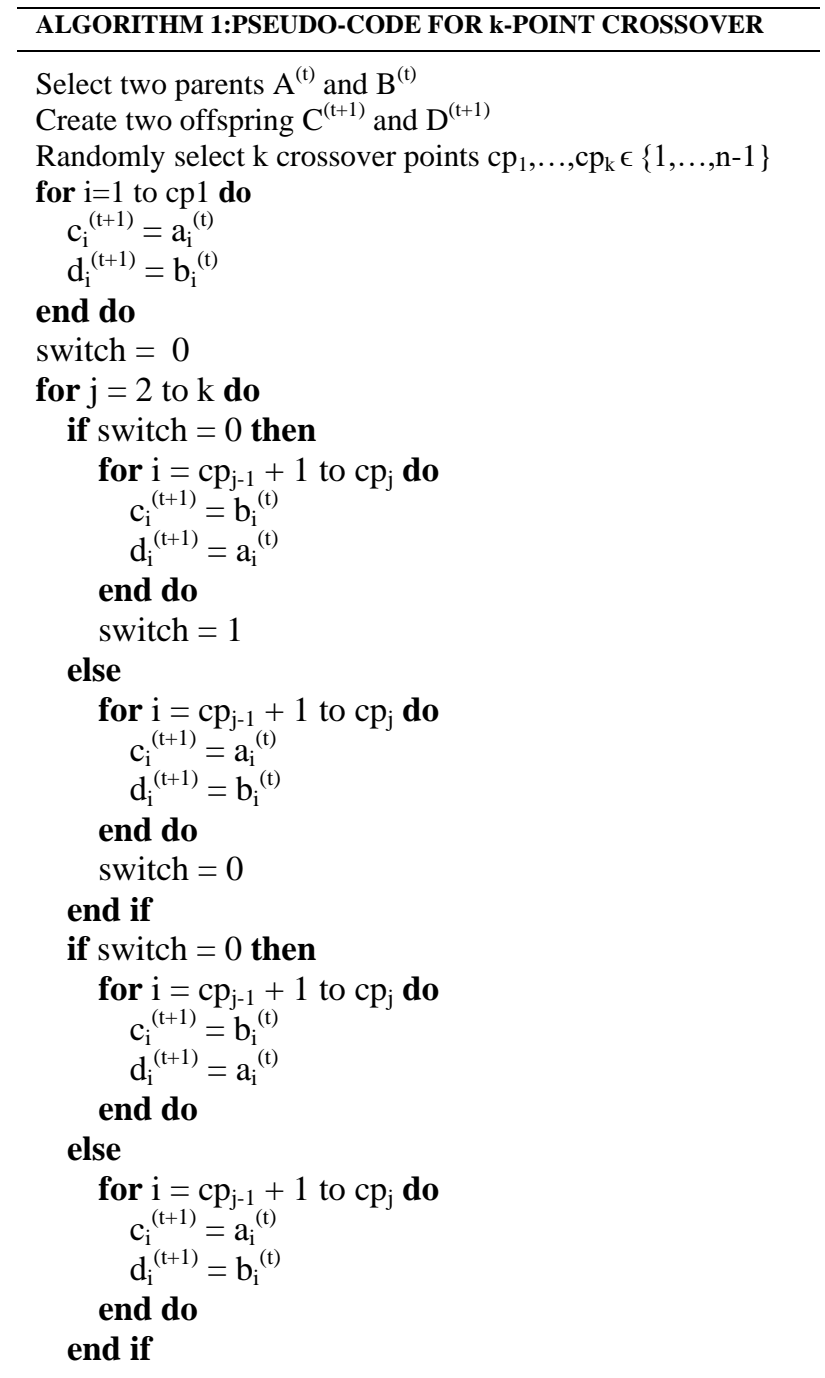

In the example shown below the points $2^{\text {nd }}$ and $3^{\text {rd }}, 5^{\text {th }}$ and $6^{\text {th }}, 8^{\text {th }}$ and $9^{\text {th }}$ and $10^{\text {th }}$ and $11^{\text {th }}$ gene are selected as crossover points where value of $k$ is 4 :

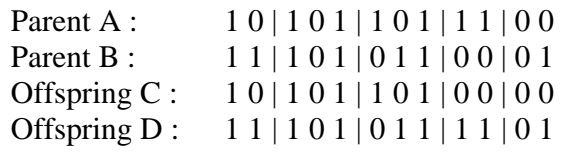

\section{A.2 Discrete TPX}

The Discrete TPX is the combination of two crossover operators, binary encoded discrete crossover and real valued three parent crossover. Using three parents for crossover will provide the operator with more exploration. The algorithm for discrete three parent crossover is given below. 


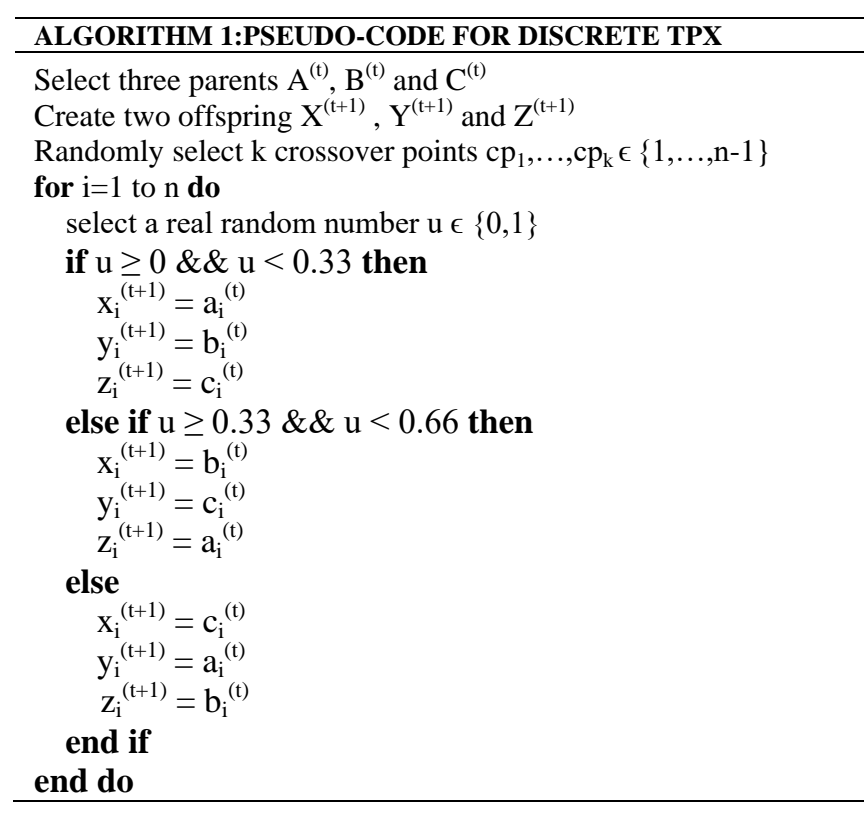

\section{B. Evolutionary Process}

The initialization of the main population with size $\mathrm{m}$ and reserve population with size $n$, fitness is calculated using their own fitness functions. As set of $\mathrm{m}$ offspring are generated by inbreeding between the parents of main population and reserve population respectively using the operators like crossover and mutation. Then $(n-m)$ offspring are generated by crossbreeding between the one parent from the main population and other from reserve population for each individual again by using crossover and mutation operator.

The newly generated individuals are evaluated using the objective function for the main population and only $\mathrm{m}$ individuals are selected among them on the basis of their fitness values for the next generation. As algorithm already has $\mathrm{m}$ offspring generated by the process of inbreeding, the crossbreed offspring can only survive if they are better than at least one of the inbreed offspring in terms of their fitness values. The newly generated individuals of the reserve population are evaluated by the fitness function of the reserve population. All of them are selected to constitute the next generation of the reserve population.

\section{RESULTS AND DISCUSSION}

Standard problems are taken for experiments from CEC'2013 Real-Parameter Optimization problem [9]. In this report, 28 benchmark functions are described. The performance of the proposed algorithm is analyzed in this section by solving the benchmark functions introduced in CEC 2013 [9]. The brief introduction to the set of problems is given as below:
Table 1. CEC'2013 Functions

\begin{tabular}{|c|c|c|}
\hline Name & Function & $\begin{array}{l}\text { Optimum } \\
\text { Value }\end{array}$ \\
\hline \multicolumn{3}{|c|}{ Unimodal Functions } \\
\hline F01 & Sphere Function & -1400 \\
\hline F02 & $\begin{array}{l}\text { Rotated High Conditioned Elliptic } \\
\text { Function }\end{array}$ & -1300 \\
\hline F03 & Rotated Bent Cigar Function & -1200 \\
\hline F04 & Rotated Discus Function & -1100 \\
\hline F05 & Different Power Function & -1000 \\
\hline \multicolumn{3}{|c|}{ Basic Multimodal Functions } \\
\hline F06 & Rotated Rosenbrock's Function & -900 \\
\hline F07 & Rotated Schaffers F7 Function & -800 \\
\hline F08 & Rotated Ackley's Function & -700 \\
\hline F09 & Rotated Weierstrass Function & -600 \\
\hline F10 & Rotated Griewank's Function & -500 \\
\hline F11 & Rastrign's Function & -400 \\
\hline F12 & Rotated Rastrign's Function & -300 \\
\hline F13 & $\begin{array}{l}\text { Non-Continuous Rotated Rastrign's } \\
\text { Function }\end{array}$ & -200 \\
\hline F14 & Schwefel's Function & -100 \\
\hline F15 & Rotated Schwefel's Function & 100 \\
\hline F16 & Rotated Katsuura Function & 200 \\
\hline F17 & Lunacek Bi_Rastrigin Function & 300 \\
\hline F18 & $\begin{array}{l}\text { Rotated Lunacek Bi_Rastrigin } \\
\text { Function }\end{array}$ & 400 \\
\hline F19 & $\begin{array}{l}\text { Expanded Griewank's plus } \\
\text { Rosenbrock's Function }\end{array}$ & 500 \\
\hline F20 & Expanded Scaffer's F6 Function & 600 \\
\hline \multicolumn{3}{|c|}{ Composition Function } \\
\hline $\mathrm{F} 21$ & $\begin{array}{l}\text { Composition Function } 1 \\
(\mathrm{n}=5, \text { Rotated })\end{array}$ & 700 \\
\hline $\mathrm{F} 22$ & $\begin{array}{l}\text { Composition Function } 2 \\
(\mathrm{n}=3, \text { Unrotated })\end{array}$ & 800 \\
\hline $\mathrm{F} 23$ & $\begin{array}{l}\text { Composition Function } 3 \\
(\mathrm{n}=3, \text { Rotated })\end{array}$ & 900 \\
\hline F24 & $\begin{array}{l}\text { Composition Function } 4 \\
(\mathrm{n}=3, \text { Rotated })\end{array}$ & 1000 \\
\hline $\mathrm{F} 25$ & $\begin{array}{l}\text { Composition Function } 5 \\
(\mathrm{n}=3, \text { Rotated })\end{array}$ & 1100 \\
\hline F26 & $\begin{array}{l}\text { Composition Function } 6 \\
(\mathrm{n}=5, \text { Rotated })\end{array}$ & 1200 \\
\hline $\mathrm{F} 27$ & $\begin{array}{l}\text { Composition Function } 7 \\
(\mathrm{n}=5, \text { Rotated })\end{array}$ & 1300 \\
\hline F28 & $\begin{array}{l}\text { Composition Function } 8 \\
(\mathrm{n}=5, \text { Rotated })\end{array}$ & 1400 \\
\hline
\end{tabular}

The results are taken on the AMD FX(tm)-8320 EightCore Processor with $3.51 \mathrm{GHz}$ clock speed. The experiments are carried on system with 16GB RAM and hard disk of capacity 500GB with operating system CentOS 6.5.

Comparison between any two algorithms is done on the basis of student $t$-test value. The $t$-test value can be calculated by using following equation 1 .

$$
t=\frac{X_{1}-X_{2}}{\sqrt{\frac{\left(n_{1}-1\right) S_{1}{ }^{2}+\left(n_{2}-1\right) S_{2}{ }^{2}}{n_{1}+n_{2}-2} \cdot\left(\frac{1}{n_{1}}+\frac{1}{n_{2}}\right)}}
$$


Where, in equation (1) $X_{1}$ and $X_{2}$ are the mean of algorithm 1 and algorithm $2, n_{1}$ and $n_{2}$ are the number of sample tested for the results, and $S_{1}$ and $S_{2}$ are the standard deviation of algorithm for a particular problem. If the value of $t$ is found to be less than 0 , will show that the first algorithm is better for solving the problem otherwise second one would be better.

\section{A. Comparison between DPGA using k-point crossover and Discrete TPX for lower dimensions}

Table 2 gives the comparative result of DPGA using $k$ point crossover versus DPGA using discrete TPX. It is clear from the $t$-test value that the Discrete TPX gives better results than $k$-point crossover for lower dimensions. Discrete TPX proves itself better for almost the functions except F17, F21, F23, F24, F25, F26 and F28 functions.

Table 2. Comparison between K-point crossover and Discrete TPX for lower dimension

\begin{tabular}{|c|c|c|c|c|c|}
\hline \multirow{2}{*}{$\begin{array}{l}\text { Fn. } \\
\text { No. }\end{array}$} & \multicolumn{2}{|c|}{ K-Point Crossover } & \multicolumn{2}{|c|}{ Discrete TPX } & \multirow{2}{*}{ t-Test } \\
\hline & $\mathbf{M}$ & SD & $\mathbf{M}$ & SD & \\
\hline $\mathrm{F} 1$ & $-1.39 \mathrm{E}+03$ & $3.66 \mathrm{E}+00$ & $-1.40 \mathrm{E}+03$ & $1.31 \mathrm{E}+00$ & 0.339491 \\
\hline $\mathrm{F} 2$ & $-4.57 \mathrm{E}+02$ & 4.03E+02 & $-9.38 \mathrm{E}+02$ & $1.68 \mathrm{E}+02$ & 0.397825 \\
\hline $\mathrm{F} 3$ & $-7.76 \mathrm{E}+02$ & $3.11 \mathrm{E}+02$ & $-8.35 \mathrm{E}+02$ & $1.25 \mathrm{E}+02$ & 0.062367 \\
\hline $\mathrm{F} 4$ & $3.24 \mathrm{E}+02$ & $7.40 \mathrm{E}+02$ & $-5.19 \mathrm{E}+02$ & $1.59 \mathrm{E}+02$ & 0.379786 \\
\hline F5 & $-9.96 \mathrm{E}+02$ & $2.90 \mathrm{E}+00$ & $-9.97 \mathrm{E}+02$ & $2.13 \mathrm{E}+00$ & 0.007903 \\
\hline F6 & $-9.00 \mathrm{E}+02$ & $2.72 \mathrm{E}-01$ & $-9.00 \mathrm{E}+02$ & $6.94 \mathrm{E}-02$ & 0.281849 \\
\hline F7 & $-7.92 \mathrm{E}+02$ & $2.21 \mathrm{E}+00$ & $-7.98 \mathrm{E}+02$ & $1.43 \mathrm{E}+00$ & 0.818658 \\
\hline F8 & $-6.95 \mathrm{E}+02$ & $2.80 \mathrm{E}+00$ & $-6.95 \mathrm{E}+02$ & $2.85 \mathrm{E}+00$ & 0.006369 \\
\hline F9 & $-5.99 \mathrm{E}+02$ & $1.47 \mathrm{E}-01$ & $-6.00 \mathrm{E}+02$ & $8.60 \mathrm{E}-02$ & 1.616449 \\
\hline F10 & $-4.98 \mathrm{E}+02$ & $1.49 \mathrm{E}+00$ & $-4.99 \mathrm{E}+02$ & $9.65 \mathrm{E}-01$ & 0.387548 \\
\hline F11 & $-3.98 \mathrm{E}+02$ & $6.99 \mathrm{E}-01$ & $-3.99 \mathrm{E}+02$ & $5.57 \mathrm{E}-01$ & 0.472732 \\
\hline F12 & $-2.98 \mathrm{E}+02$ & $6.52 \mathrm{E}-01$ & $-3.00 \mathrm{E}+02$ & $3.23 \mathrm{E}-01$ & 1.151011 \\
\hline F13 & $-1.97 \mathrm{E}+02$ & $1.12 \mathrm{E}+00$ & $-2.00 \mathrm{E}+02$ & $0.00 \mathrm{E}+00$ & 0.756002 \\
\hline F14 & $-8.87 \mathrm{E}+01$ & $9.77 \mathrm{E}+00$ & $-9.11 \mathrm{E}+01$ & $5.60 \mathrm{E}+00$ & 0.083637 \\
\hline F15 & $1.13 \mathrm{E}+02$ & $7.41 \mathrm{E}+00$ & $1.08 \mathrm{E}+02$ & $5.94 \mathrm{E}+00$ & 0.201622 \\
\hline F16 & $2.03 \mathrm{E}+02$ & $1.82 \mathrm{E}+00$ & $2.00 \mathrm{E}+02$ & $0.00 \mathrm{E}+00$ & 0.468688 \\
\hline F17 & $3.03 \mathrm{E}+02$ & $6.07 \mathrm{E}-01$ & $3.03 \mathrm{E}+02$ & 4.43E-01 & -0.0237 \\
\hline F18 & $4.04 \mathrm{E}+02$ & $8.73 \mathrm{E}-01$ & $4.03 \mathrm{E}+02$ & $6.82 \mathrm{E}-01$ & 0.082222 \\
\hline F19 & $5.00 \mathrm{E}+02$ & $2.84 \mathrm{E}-01$ & $5.00 \mathrm{E}+02$ & $5.78 \mathrm{E}-02$ & 0.43412 \\
\hline $\mathrm{F} 20$ & $6.00 \mathrm{E}+02$ & $1.89 \mathrm{E}-01$ & $6.00 \mathrm{E}+02$ & $8.80 \mathrm{E}-02$ & 0.589632 \\
\hline F21 & $7.25 \mathrm{E}+02$ & $1.88 \mathrm{E}+01$ & $7.65 \mathrm{E}+02$ & $1.47 \mathrm{E}+01$ & -0.69501 \\
\hline $\mathrm{F} 22$ & $8.31 \mathrm{E}+02$ & $1.75 \mathrm{E}+01$ & $8.22 \mathrm{E}+02$ & 7.97E+00 & 0.167756 \\
\hline $\mathrm{F} 23$ & $9.44 \mathrm{E}+02$ & $3.44 \mathrm{E}+01$ & $9.84 \mathrm{E}+02$ & $2.29 \mathrm{E}+01$ & -0.38725 \\
\hline F24 & $1.01 \mathrm{E}+03$ & $2.19 \mathrm{E}+00$ & $1.02 \mathrm{E}+03$ & $7.88 \mathrm{E}+00$ & -1.96993 \\
\hline F25 & $1.12 \mathrm{E}+03$ & $1.29 \mathrm{E}+01$ & $1.17 \mathrm{E}+03$ & $3.17 \mathrm{E}+01$ & -1.17709 \\
\hline F26 & $1.20 \mathrm{E}+03$ & $2.88 \mathrm{E}+00$ & $1.21 \mathrm{E}+03$ & $2.62 \mathrm{E}+00$ & -0.26336 \\
\hline F27 & $1.43 \mathrm{E}+03$ & $3.41 \mathrm{E}+01$ & $1.40 \mathrm{E}+03$ & $2.16 \mathrm{E}+01$ & 0.295389 \\
\hline F28 & $1.45 \mathrm{E}+03$ & $2.53 \mathrm{E}+01$ & $1.48 \mathrm{E}+03$ & $2.43 \mathrm{E}+01$ & -0.43603 \\
\hline
\end{tabular}

B. Comparison between DPGA using k-point crossover and PSO

Table 3 shows the comparison of the results of DPGA using $k$-point crossover versus DPGA using discrete TPX. For higher dimensions the $k$-point crossover is found to be better than proposed discrete TPX except F2, F4, F14, F15, F16, F22, F24, F26 and F26.

Table 3. Comparison between K-point crossover and Discrete TPX for higher dimension

\begin{tabular}{|c|c|c|c|c|c|}
\hline \multirow{2}{*}{$\begin{array}{l}\text { Fn. } \\
\text { No. }\end{array}$} & \multicolumn{2}{|c|}{ K-Point Crossover } & \multicolumn{2}{|c|}{ Discrete TPX } & \multirow{2}{*}{ t-Test } \\
\hline & $\mathbf{M}$ & SD & $\mathbf{M}$ & SD & \\
\hline $\mathrm{F} 1$ & $1.50 \mathrm{E}+05$ & $1.21 \mathrm{E}+04$ & $1.57 \mathrm{E}+05$ & $1.20 \mathrm{E}+04$ & -0.19349 \\
\hline $\mathrm{F} 2$ & $5.83 \mathrm{E}+09$ & $7.36 \mathrm{E}+08$ & $5.19 \mathrm{E}+09$ & $1.33 \mathrm{E}+09$ & 0.286763 \\
\hline $\mathrm{F} 3$ & $9.84 \mathrm{E}+18$ & $1.40 \mathrm{E}+19$ & $1.29 \mathrm{E}+20$ & $2.04 \mathrm{E}+20$ & -1.54493 \\
\hline $\mathrm{F} 4$ & $4.73 E+09$ & $2.36 \mathrm{E}+09$ & $4.23 \mathrm{E}+05$ & $1.55 \mathrm{E}+05$ & 0.668036 \\
\hline F5 & $6.35 \mathrm{E}+04$ & $1.44 \mathrm{E}+04$ & $6.88 \mathrm{E}+04$ & $1.91 \mathrm{E}+04$ & -0.1223 \\
\hline F6 & $2.28 \mathrm{E}+04$ & $3.11 \mathrm{E}+03$ & $2.86 \mathrm{E}+04$ & $4.21 \mathrm{E}+03$ & -0.61451 \\
\hline F7 & $3.27 \mathrm{E}+06$ & $2.51 \mathrm{E}+06$ & $3.40 \mathrm{E}+06$ & $2.88 \mathrm{E}+06$ & -0.01785 \\
\hline F8 & $-6.83 E+02$ & $4.82 \mathrm{E}+00$ & $-6.79 \mathrm{E}+02$ & $6.62 \mathrm{E}-02$ & -0.27857 \\
\hline F9 & $-5.43 \mathrm{E}+02$ & $3.04 \mathrm{E}+01$ & $-5.19 \mathrm{E}+02$ & $1.94 \mathrm{E}+00$ & -0.26418 \\
\hline F10 & $2.24 \mathrm{E}+04$ & $2.83 \mathrm{E}+03$ & $2.36 \mathrm{E}+04$ & $2.75 \mathrm{E}+03$ & -0.14357 \\
\hline F11 & $2.08 \mathrm{E}+03$ & $3.14 \mathrm{E}+02$ & $2.21 \mathrm{E}+03$ & $1.51 \mathrm{E}+02$ & -0.1364 \\
\hline $\mathrm{F} 12$ & $1.99 \mathrm{E}+03$ & $1.67 \mathrm{E}+02$ & $2.08 \mathrm{E}+03$ & $2.39 \mathrm{E}+02$ & -0.19098 \\
\hline F13 & $2.04 \mathrm{E}+03$ & $1.66 \mathrm{E}+02$ & $2.20 \mathrm{E}+03$ & $1.60 \mathrm{E}+02$ & -0.3274 \\
\hline F14 & $1.60 \mathrm{E}+04$ & $5.63 \mathrm{E}+02$ & $1.58 \mathrm{E}+04$ & $3.07 \mathrm{E}+02$ & 0.105333 \\
\hline $\mathrm{F} 15$ & $1.70 \mathrm{E}+04$ & $3.34 \mathrm{E}+02$ & $1.66 \mathrm{E}+04$ & $6.26 \mathrm{E}+02$ & 0.377395 \\
\hline F16 & $2.07 \mathrm{E}+02$ & 7.34E-01 & $2.00 \mathrm{E}+02$ & $0.00 \mathrm{E}+00$ & 3.08635 \\
\hline F17 & $4.92 \mathrm{E}+03$ & $4.85 \mathrm{E}+02$ & $5.45 \mathrm{E}+03$ & $3.35 \mathrm{E}+02$ & -0.36572 \\
\hline F18 & $5.16 \mathrm{E}+03$ & $4.01 \mathrm{E}+02$ & $5.23 \mathrm{E}+03$ & $5.40 \mathrm{E}+02$ & -0.05528 \\
\hline F19 & $2.04 \mathrm{E}+07$ & $5.98 \mathrm{E}+06$ & $2.50 \mathrm{E}+07$ & $4.12 \mathrm{E}+06$ & -0.25786 \\
\hline F20 & $6.25 \mathrm{E}+02$ & $0.00 \mathrm{E}+00$ & $6.25 \mathrm{E}+02$ & $0.00 \mathrm{E}+00$ & $\operatorname{Inf}$ \\
\hline $\mathrm{F} 21$ & $1.23 \mathrm{E}+04$ & $6.12 \mathrm{E}+02$ & $1.31 \mathrm{E}+04$ & $8.44 \mathrm{E}+02$ & -0.41176 \\
\hline $\mathrm{F} 22$ & $1.79 \mathrm{E}+04$ & $4.27 \mathrm{E}+02$ & $1.78 \mathrm{E}+04$ & $4.21 \mathrm{E}+02$ & 0.065736 \\
\hline $\mathrm{F} 23$ & $1.80 \mathrm{E}+04$ & $2.53 \mathrm{E}+02$ & $1.80 \mathrm{E}+04$ & $2.86 \mathrm{E}+02$ & -0.07589 \\
\hline $\mathrm{F} 24$ & $1.52 \mathrm{E}+03$ & $9.39 \mathrm{E}+01$ & $1.48 \mathrm{E}+03$ & $4.95 \mathrm{E}+01$ & 0.116111 \\
\hline F25 & $1.51 \mathrm{E}+03$ & $6.19 \mathrm{E}+00$ & $1.51 \mathrm{E}+03$ & $5.90 \mathrm{E}+00$ & -0.04611 \\
\hline F26 & $1.73 \mathrm{E}+03$ & $1.08 \mathrm{E}+01$ & $1.72 \mathrm{E}+03$ & $7.90 \mathrm{E}+00$ & 0.159713 \\
\hline $\mathrm{F} 27$ & $3.93 \mathrm{E}+03$ & $6.03 \mathrm{E}+01$ & $3.87 \mathrm{E}+03$ & $8.80 \mathrm{E}+01$ & 0.381049 \\
\hline F28 & $1.64 \mathrm{E}+04$ & $1.56 \mathrm{E}+03$ & $1.69 \mathrm{E}+04$ & $1.71 \mathrm{E}+03$ & -0.10427 \\
\hline
\end{tabular}

C. Comparison between DPGA using k-point crossover and PSO

Table 4 shows the results of comparison between PSO and DPGA using $k$-point crossover. The $k$-point crossover is found to be good for only F8 function. For all the other functions SPSO is better. 
Table 4.Comparison between PSO[7] and DPGA using k-point crossover

\begin{tabular}{|c|c|c|c|c|c|}
\hline \multirow{2}{*}{$\begin{array}{l}\text { Fn. } \\
\text { No. }\end{array}$} & \multicolumn{2}{|c|}{ PSO [7] } & \multicolumn{2}{|c|}{ K-Point Crossover } & \multirow{2}{*}{ t-Test } \\
\hline & $\mathbf{M}$ & SD & $\mathbf{M}$ & SD & \\
\hline $\mathrm{F} 1$ & $-1.40 \mathrm{E}+03$ & $3.18 \mathrm{E}-13$ & $1.50 \mathrm{E}+05$ & $1.21 \mathrm{E}+04$ & -92.4752 \\
\hline $\mathrm{F} 2$ & $6.79 \mathrm{E}+05$ & $1.87 \mathrm{E}+05$ & $5.83 \mathrm{E}+09$ & $7.36 \mathrm{E}+08$ & -58.6657 \\
\hline F3 & $4.37 \mathrm{E}+08$ & $9.47 \mathrm{E}+08$ & $9.84 \mathrm{E}+18$ & $1.40 \mathrm{E}+19$ & -5.1836 \\
\hline $\mathrm{F} 4$ & $4.99 \mathrm{E}+04$ & $8.72 \mathrm{E}+03$ & $4.73 \mathrm{E}+09$ & $2.36 \mathrm{E}+09$ & -14.8382 \\
\hline F5 & $-1.00 \mathrm{E}+03$ & $5.41 \mathrm{E}-05$ & $6.35 \mathrm{E}+04$ & $1.44 \mathrm{E}+04$ & -33.2812 \\
\hline F6 & $-8.57 \mathrm{E}+02$ & $2.41 \mathrm{E}+01$ & $2.28 \mathrm{E}+04$ & $3.11 \mathrm{E}+03$ & -52.2599 \\
\hline F7 & $-7.14 \mathrm{E}+02$ & $1.53 \mathrm{E}+01$ & $3.27 \mathrm{E}+06$ & $2.51 \mathrm{E}+06$ & -9.64366 \\
\hline F8 & $-6.79 E+02$ & $4.25 \mathrm{E}-02$ & $-6.83 E+02$ & $4.82 \mathrm{E}+00$ & 5.279006 \\
\hline F9 & $-5.46 \mathrm{E}+02$ & $6.74 \mathrm{E}+00$ & $-5.43 \mathrm{E}+02$ & $3.04 \mathrm{E}+01$ & -0.06841 \\
\hline F10 & $-5.00 \mathrm{E}+02$ & $2.38 \mathrm{E}-01$ & $2.24 \mathrm{E}+04$ & $2.83 \mathrm{E}+03$ & -59.7295 \\
\hline F11 & $-1.70 \mathrm{E}+02$ & 4.18E+01 & $2.08 \mathrm{E}+03$ & $3.14 \mathrm{E}+02$ & -7.54086 \\
\hline $\mathrm{F} 12$ & $-6.52 \mathrm{E}+01$ & $4.87 \mathrm{E}+01$ & $1.99 \mathrm{E}+03$ & $1.67 \mathrm{E}+02$ & -5.94846 \\
\hline F13 & $2.28 \mathrm{E}+02$ & $6.22 \mathrm{E}+01$ & $2.04 \mathrm{E}+03$ & $1.66 \mathrm{E}+02$ & -4.10339 \\
\hline F14 & $7.16 \mathrm{E}+03$ & $8.53 \mathrm{E}+02$ & $1.60 \mathrm{E}+04$ & $5.63 \mathrm{E}+02$ & -1.46975 \\
\hline F15 & $8.02 \mathrm{E}+03$ & $1.14 \mathrm{E}+03$ & $1.70 \mathrm{E}+04$ & $3.34 \mathrm{E}+02$ & -1.10923 \\
\hline F16 & $2.02 \mathrm{E}+02$ & $3.87 \mathrm{E}-01$ & $2.07 \mathrm{E}+02$ & 7.34E-01 & -1.7531 \\
\hline F17 & $6.11 \mathrm{E}+02$ & $6.62 \mathrm{E}+01$ & $4.92 \mathrm{E}+03$ & $4.85 \mathrm{E}+02$ & -9.12257 \\
\hline F18 & $6.91 \mathrm{E}+02$ & $6.24 \mathrm{E}+01$ & $5.16 \mathrm{E}+03$ & $4.01 \mathrm{E}+02$ & -10.0598 \\
\hline F19 & $5.37 \mathrm{E}+02$ & $1.20 \mathrm{E}+01$ & $2.04 \mathrm{E}+07$ & $5.98 \mathrm{E}+06$ & -25.1922 \\
\hline F20 & $6.23 \mathrm{E}+02$ & $1.19 \mathrm{E}+00$ & $6.25 \mathrm{E}+02$ & $0.00 \mathrm{E}+00$ & -0.27242 \\
\hline F21 & $1.54 \mathrm{E}+03$ & $3.04 \mathrm{E}+02$ & $1.23 \mathrm{E}+04$ & $6.12 \mathrm{E}+02$ & -5.01516 \\
\hline F22 & $9.72 \mathrm{E}+03$ & $1.40 \mathrm{E}+03$ & $1.79 \mathrm{E}+04$ & $4.27 \mathrm{E}+02$ & -0.82251 \\
\hline $\mathrm{F} 23$ & $1.13 \mathrm{E}+04$ & $1.35 \mathrm{E}+03$ & $1.80 \mathrm{E}+04$ & $2.53 \mathrm{E}+02$ & -0.70149 \\
\hline F24 & $1.34 \mathrm{E}+03$ & $1.69 \mathrm{E}+01$ & $1.52 \mathrm{E}+03$ & $9.39 \mathrm{E}+01$ & -1.44154 \\
\hline F25 & $1.50 \mathrm{E}+03$ & $2.05 \mathrm{E}+01$ & $1.51 \mathrm{E}+03$ & $6.19 \mathrm{E}+00$ & -0.07841 \\
\hline F26 & $1.63 \mathrm{E}+03$ & $9.06 \mathrm{E}+01$ & $1.73 \mathrm{E}+03$ & $1.08 \mathrm{E}+01$ & -0.15822 \\
\hline $\mathrm{F} 27$ & $2.98 \mathrm{E}+03$ & $1.64 \mathrm{E}+02$ & $3.93 \mathrm{E}+03$ & $6.03 \mathrm{E}+01$ & -0.82792 \\
\hline F28 & $1.80 \mathrm{E}+03$ & $1.30 \mathrm{E}+03$ & $1.64 \mathrm{E}+04$ & $1.56 \mathrm{E}+03$ & -1.57995 \\
\hline
\end{tabular}

D. Comparison between DPGA using Discrete TPX and PSO [7]

Table 5 represents the comparison between the results of DPGA using discrete TPX and the PSO [7]. Discrete TPX is found to be good for only F16 function. For all the other functions the PSO is better than DPGA.

Table 5. Comparison between DPGA using Discrete TPX and PSO

\begin{tabular}{|c|c|c|c|c|c|}
\hline \multirow{2}{*}{$\begin{array}{c}\text { Fn. } \\
\text { No. }\end{array}$} & \multicolumn{2}{|c|}{ PSO [7] } & \multicolumn{2}{c|}{ Discrete TPX } & \multirow{2}{*}{ t-Test } \\
\cline { 2 - 5 } & M & SD & M & SD & \\
\hline F1 & $-1.40 \mathrm{E}+03$ & $3.18 \mathrm{E}-13$ & $1.57 \mathrm{E}+05$ & $1.20 \mathrm{E}+04$ & -97.669 \\
\hline F2 & $6.79 \mathrm{E}+05$ & $1.87 \mathrm{E}+05$ & $5.19 \mathrm{E}+09$ & $1.33 \mathrm{E}+09$ & -28.9258 \\
\hline F3 & $4.37 \mathrm{E}+08$ & $9.47 \mathrm{E}+08$ & $1.29 \mathrm{E}+20$ & $2.04 \mathrm{E}+20$ & -4.67981 \\
\hline
\end{tabular}

\begin{tabular}{|c|c|c|c|c|c|}
\hline $\mathrm{F} 4$ & $4.99 \mathrm{E}+04$ & $8.72 \mathrm{E}+03$ & $4.23 \mathrm{E}+05$ & $1.55 \mathrm{E}+05$ & -5.72792 \\
\hline F5 & $-1.00 \mathrm{E}+03$ & $5.41 \mathrm{E}-05$ & $6.88 \mathrm{E}+04$ & $1.91 \mathrm{E}+04$ & -27.046 \\
\hline F6 & $-8.57 \mathrm{E}+02$ & $2.41 \mathrm{E}+01$ & $2.86 \mathrm{E}+04$ & $4.21 \mathrm{E}+03$ & -49.7189 \\
\hline F7 & $-7.14 \mathrm{E}+02$ & $1.53 \mathrm{E}+01$ & $3.40 \mathrm{E}+06$ & $2.88 \mathrm{E}+06$ & -8.74376 \\
\hline F8 & $-6.79 E+02$ & $4.25 \mathrm{E}-02$ & $-6.79 \mathrm{E}+02$ & $6.62 \mathrm{E}-02$ & -0.80331 \\
\hline F9 & $-5.46 \mathrm{E}+02$ & $6.74 \mathrm{E}+00$ & $-5.19 \mathrm{E}+02$ & $1.94 \mathrm{E}+00$ & -0.57387 \\
\hline F10 & $-5.00 \mathrm{E}+02$ & $2.38 \mathrm{E}-01$ & $2.36 \mathrm{E}+04$ & $2.75 \mathrm{E}+03$ & -64.9629 \\
\hline F11 & $-1.70 \mathrm{E}+02$ & $4.18 \mathrm{E}+01$ & $2.21 \mathrm{E}+03$ & $1.51 \mathrm{E}+02$ & -8.03473 \\
\hline F12 & $-6.52 \mathrm{E}+01$ & $4.87 \mathrm{E}+01$ & $2.08 \mathrm{E}+03$ & $2.39 \mathrm{E}+02$ & -6.21523 \\
\hline F13 & $2.28 \mathrm{E}+02$ & $6.22 \mathrm{E}+01$ & $2.20 \mathrm{E}+03$ & $1.60 \mathrm{E}+02$ & -4.47684 \\
\hline F14 & $7.16 \mathrm{E}+03$ & $8.53 \mathrm{E}+02$ & $1.58 \mathrm{E}+04$ & $3.07 \mathrm{E}+02$ & -1.44028 \\
\hline F15 & $8.02 \mathrm{E}+03$ & $1.14 \mathrm{E}+03$ & $1.66 \mathrm{E}+04$ & $6.26 \mathrm{E}+02$ & -1.06132 \\
\hline F16 & $2.02 \mathrm{E}+02$ & $3.87 \mathrm{E}-01$ & $2.00 \mathrm{E}+02$ & $0.00 \mathrm{E}+00$ & 0.731805 \\
\hline F17 & $6.11 \mathrm{E}+02$ & $6.62 \mathrm{E}+01$ & $5.45 \mathrm{E}+03$ & $3.35 \mathrm{E}+02$ & -10.3047 \\
\hline F18 & $6.91 \mathrm{E}+02$ & $6.24 \mathrm{E}+01$ & $5.23 \mathrm{E}+03$ & $5.40 \mathrm{E}+02$ & -10.15 \\
\hline F19 & $5.37 \mathrm{E}+02$ & $1.20 \mathrm{E}+01$ & $2.50 \mathrm{E}+07$ & $4.12 \mathrm{E}+06$ & -44.8977 \\
\hline F20 & $6.23 \mathrm{E}+02$ & $1.19 \mathrm{E}+00$ & $6.25 \mathrm{E}+02$ & $0.00 \mathrm{E}+00$ & -0.27242 \\
\hline F21 & $1.54 \mathrm{E}+03$ & $3.04 \mathrm{E}+02$ & $1.31 \mathrm{E}+04$ & $8.44 \mathrm{E}+02$ & -5.36642 \\
\hline F22 & $9.72 \mathrm{E}+03$ & $1.40 \mathrm{E}+03$ & $1.78 \mathrm{E}+04$ & $4.21 \mathrm{E}+02$ & -0.81399 \\
\hline F23 & $1.13 \mathrm{E}+04$ & $1.35 \mathrm{E}+03$ & $1.80 \mathrm{E}+04$ & $2.86 \mathrm{E}+02$ & -0.70756 \\
\hline F24 & $1.34 \mathrm{E}+03$ & $1.69 \mathrm{E}+01$ & $1.48 \mathrm{E}+03$ & $4.95 \mathrm{E}+01$ & -1.1733 \\
\hline F25 & $1.50 \mathrm{E}+03$ & $2.05 \mathrm{E}+01$ & $1.51 \mathrm{E}+03$ & $5.90 \mathrm{E}+00$ & -0.08436 \\
\hline F26 & $1.63 \mathrm{E}+03$ & $9.06 \mathrm{E}+01$ & $1.72 \mathrm{E}+03$ & $7.90 \mathrm{E}+00$ & -0.1501 \\
\hline F27 & $2.98 \mathrm{E}+03$ & $1.64 \mathrm{E}+02$ & $3.87 \mathrm{E}+03$ & $8.80 \mathrm{E}+01$ & -0.76773 \\
\hline F28 & $1.80 \mathrm{E}+03$ & $1.30 \mathrm{E}+03$ & $1.69 \mathrm{E}+04$ & $1.71 \mathrm{E}+03$ & -1.63318 \\
\hline
\end{tabular}

\section{CONCLUSION}

DPGA is a diversity based technique using two populations. Two crossover operators are experimented on CEC'2013 problem set. DPGA can successfully solve the CEC'2013 problems with smaller dimensions but it is observed that the algorithm is suffering by the curse of dimensionality i.e. as the dimension increases from 2 to 50 the algorithm loses its accuracy to find the optimum solution.

DPGA using discrete TPX and it found better than kpoint crossover only for the problems having lower dimensions. But for higher dimension problems k-point crossover is able to maintain consistency for obtaining an optimal solution. On the basis of t-test evaluation, the results of both types of the crossover are also compared with the results of PSO on the same problem. PSO has found better than DPGA to solve the functions of CEC'2013.

In the future, the results of the DPGA algorithm for CEC'2013 could be improved by using better survival selection and crossover, mutation operators.

Further, DPGA performance can be improved by adding more reserve populations. A performance 
comparison of DPGA could be done with other metaheuristic. DPGA can be tested on the latest test bed of CEC.

\section{ACKNOWLEDGMENT}

We express our sincere thanks to all the authors, whose papers in the area of Dual Population EA and published in various conference proceedings and journals.

\section{REFERENCES}

[1] T. Park, K. Ruy, "A Dual-Population Genetic Algorithm for Adaptive Diversity Control", IEEE transactions on evolutionary computation, vol. 14, no. 6,2010 DOI:10.1109/TEVC.2010.2043362.

[2] A.J. Umbarkar, M.S. Joshi, P.D. Sheth, "Diversity-Based Dual-Population Genetic Algorithm (DPGA): A Review" In Proceedings of Fourth International Conference on Soft Computing for Problem Solving, (AISC, vol 335). 2015. DOI https://doi.org/10.1007/978-81-322-2217-0_19

[3] T. Park and K. Ruy, "A dual population genetic algorithm with evolving diversity", in Proc. IEEE Congr. Evol. Comput., pp. 3516-3522, 2007. DOI: 10.1109/CEC.2007.4424928.

[4] T. Park and K. Ruy, "Adjusting population distance for dual-population genetic algorithm," in Proc. of Aust. Joint Conf. Artif. Intell., pp. 171-180, 2007. DOI: 10.1109/TEVC.2010.2043362.

[5] T. Park, R. Choe, K. Ruy, "Dual-population Genetic Algorithm for Nonstationary Optimization", in Proc. GECCO'08 ACM, 2008, pp.1025-1032. DOI: $10.1145 / 1389095.1389286$

[6] A. Umbarkar, M. Joshi, "Dual Population Genetic Algorithm (GA) versus OpenMP GA for Multimodal Function Optimization", International Journal of Computer Applications, 64(19):29-36, February 2013. DOI: 10.5120/10744-5516.

[7] M. Zambrano-Bigiarini, M. Clerc and R. Rojas, "Standard Particle Swarm Optimisation 2011 at CEC-2013: A baseline for future PSO improvements," Evolutionary Computation (CEC), 2013 IEEE Congress on, vol., no., pp.2337,2344, 20-23 June 2013. doi: 10.1109/CEC.2013.6557848.

[8] M. Mehra, M. Jayalal, A. John Arul, S. Rajeswari, K. Kuriakose, S. Murty, "Study on Different Crossover Mechanisms of Genetic Algorithm for Test Interval Optimization for Nuclear Power Plants", IJISA, vol.6, no.1, 2014, pp.20-28. DOI: 10.5815/ijisa.2014.01.03.

[9] J. Liang, B. Qu, P. Suganthan, A. Hernández-Díaz, "Problem definitions and evaluation criteria for the CEC 2013 special session on real-parameter optimization", Computational Intelligence Laboratory, Zhengzhou University, Zhengzhou, China and Nanyang Technological University, Singapore, Technical Report 2013, vol. 201212.
[10] S. Elsayed, R. Sarker and D. Essam, "A genetic algorithm for solving the CEC'2013 competition problems on realparameter optimization," Evolutionary Computation (CEC), 2013 IEEE Congress on, vol., no., pp.356,360, 20-23 June 2013. DOI: 10.1109/CEC.2013.6557591.

[11] C. Worasucheep, "A Particle Swarm Optimization with Diversity-Guided Convergence Acceleration and Stagnation Avoidance", in Proc. of 8th International Conference on Natural Computation (ICNC 2012), pp.733-738, 2012. DOI: 10.1109/ICNC.2012.6234647.

[12] B. Qu, P. Suganthan, "Constrained Multi-Objective Optimization Algorithm with Diversity Enhanced Differential Evolution", IEEE conference on evolutionary $\begin{array}{lll}\text { computation } & \text { (CEC), } & 2010 .\end{array}$ 10.1109/CEC.2010.5585947.

\section{Authors' Profiles}

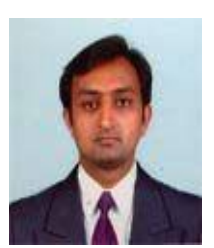

A. J. Umbarkar is presently working as an Assistant Professor in Information Technology at Walchand College of Engineering, at Sangli, MS, India. He has received his Bachelor of Engineering $(\mathrm{BE})$ in Computer Engineering from PICT, Pune, MS India, his Master of Engineering (ME) from Computer Science and Engineering (CSE) from Walchand College of Engineering, at Sangli, MS, India and received $\mathrm{PhD}$ from Government College Engineering, Aurangabad, from University: BAMU Aurangabad, MS, India. He has 15 years of teaching experience. His research interests include Parallel Genetic Algorithms, Parallel Evolutionary Algorithms and Parallel programming. He has published about 32 research papers in Conferences and Journals.

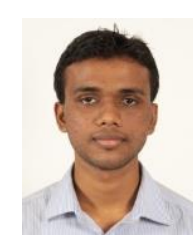

Latik R. Moon has completed Bachelor of Technology (B. Tech. 2013) in Computer Science and Engineering from SGGSIE\&T, Nanded, MS, India; and Master of Technology (M. Tech. 2015) in Information Technology from Walchand College of Engineering, Sangli, MS, India. His research interests are Parallel Programming, High Performance Computing, GPU Programming and Evolutionary Algorithms.

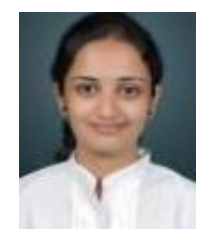

Pranali D. Sheth has completed Bachelor of Technology (B. Tech- 2007) and Master of Technology (M. Tech - 2014) in Information Technology from Walchand College of Engineering, Sangli, MS, India. Her research interests are Parallel Evolutionary Programming, High Performance Computing, Data Mining and Image Processing.

How to cite this paper: A. J. Umbarkar, L. R. Moon, P. D. Sheth," Comparative Study of CEC'2013 Problem Using Dual Population Genetic Algorithm", International Journal of Information Engineering and Electronic Business(IJIEEB), Vol.10, No.5, pp. 40-45, 2018. DOI: 10.5815/ijieeb.2018.05.06 